\title{
A CHARACTERIZATION OF $C(X)$ FOR LOCALLY CONNECTED $X^{1}$
}

\author{
DANIEL E. WULBERT
}

Introduction. The objective of this paper is to present a normed linear lattice characterization of $C(X)$ for completely regular spaces $X$ which have locally connected Stone-Cech compactifications. Characterizations of $C(X)$ for completely regular spaces $X$ which have disconnected, basically disconnected, and extremally disconnected Stone-Cech compactifications are known, and can be found in [4]. The characterization we present provides elementary proofs for several known results concerning locally connected compactifications. These include the theorem due to Banaschewski (for the sufficiency) and Henriksen and Isbell (for the necessity) that a completely regular space has a locally connected Stone-Cech compactification if and only if it is locally connected and pseudocompact.

All topological spaces considered in this paper are assumed to be completely regular. Normed linear lattices are of ten called normed vector lattices in the literature. No distinction is intended here.

An announcement of this paper containing the characterization lemma was presented at the International Symposium on Extension Theory of Topological Structures, held in Berlin in August 1967, and will appear in the proceedings of this Symposium.

The key lemma for the proof of Theorem 3 was established by Professor A. J. Ward and is presented here (Lemma 5) essentially without change. Professor Ward also supplied a fundamental counterexample presented here (Example 9). It is a pleasure to acknowledge Professor Ward's assistance.

The characterization lemma and applications to locally connected compactifications. Let $E$ be a normed linear lattice. A subset $\left\{p_{i}: i=1,2, \cdots, n\right\}$ of $E$ is a decomposition of a point $x$ in $E$ if $\left|p_{i}\right| \wedge\left|p_{j}\right|=0$ for $i \neq j$ and $\sum_{i=1}^{n} p_{i}=x$. A member $x$ of $E$ is well behaved if for each $r>0, x$ admits a decomposition $\left\{p_{i}: i=1,2, \cdots, n\right\}$ such that for $i=1,2, \cdots, n-1, p_{i}$ admits no proper decomposition and $\left\|p_{n}\right\|<r$. If every point in $E$ is well behaved, we say that $E$ is well behaved.

Let $X$ be a completely regular space and let $C(X)$ denote the

Received by the editors March 7, 1968.

${ }_{1}^{1}$ This research was partially supported by National Science Foundation Grant No. GP-8175. 
normed linear lattice of all bounded real-valued continuous functions on $X$ with the supremum norm and the usual pointwise operations.

1. Characterization Lemma. The normed linear lattice $C(X)$ is well behaved if and only if $X$ is pseudocompact and locally connected.

Proof. Suppose $C(X)$ is a well behaved normed linear lattice. We first show that $X$ is pseudocompact. If $X$ is not pseudocompact then $C(X)$ contains an isometric lattice isomorphic copy of $(m)$, the space of all bounded sequences. Since $(m)$ is clearly not well behaved $C(X)$ cannot be well behaved.

We next show that $X$ is locally connected. Let $U$ be a neighborhood of a point $x$ in $X$. Since $C(X)$ is well behaved there is a continuous function $f$ of norm 1 which achieves its norm at $x$, vanishes off $U$, and admits no proper decomposition. It follows that $\{y$ in $X: f(y) \neq 0\}$ is a connected neighborhood of $x$ contained in $U$.

Now suppose $X$ is locally connected and pseudocompact, we will show that $C(X)$ is well behaved. Let $f$ be an arbitrary function in $C(X)$. Since $X$ is locally connected the components of the open set

$$
B=\{x \text { in } X: f(x) \neq 0\}
$$

are open. For any positive $r$ the family of open sets

$$
F=\{G \cap\{x \text { in } X:|f(x)|>r\}: G \text { a component of } B\}
$$

has the property that every $x$ in $X$ has a neighborhood which meets at most one member of $F$. Since $X$ is pseudocompact, $F$ can contain only a finite number of nonempty sets. It follows that $f$-and thus $C(X)$-is well behaved. This completes the proof.

The following is a direct result of the lemma.

2. ThEOREM. The Stone-Čech compactifications of a completely regular space $X$ is locally connected if and only if $X$ is pseudocompact and locally connected.

Let $K_{1} X$ and $K_{2} X$ be compactifications of $X$. If for every continuous function $f$ in $D\left(K_{1} X\right)$ there is a function $h$ in $C\left(K_{2} X\right)$ which agrees with $f$ on $X$ we will write $K_{2} X \geqq K_{1} X$. It is obvious from the theorem that $K_{1} X$ is locally connected, if and only if the subspace of $C(X)$ consisting of functions which have extensions to continuous functions on $K_{1} X$ is a well behaved linear lattice.

3. THEOREM. If $K_{2} X \geqq K_{1} X$, and $K_{2} X$ is locally connected then $K_{1} X$ is locally connected. 
The theorem follows from the observation preceding the statement of the theorem and from Lemmas 4, 5, 6, 7 and 8 below. The lemmas relate properties of decompositions of a function to properties of the support of the function.

Let $X$ be a completely regular space, and let $f$ be in $C(X)$. Define

$$
B(f)=\{x \text { in } X: f(x) \neq 0\} .
$$

For $r>0$ define

$$
F(f, r)=\{G \cap\{x \text { in } X:|f(x)|>r\}: G \text { a component of } B(f)\} .
$$

4. LEMMA. The function $f$ admits no proper decomposition if and only if $B(f)$ is connected.

5. Lemma (A. J. WARD). If, for some $r>0, F(f, r)$ has an infinite number of members, then there is an $r^{\prime}>0$ with the following property. For each integer $k$ there is a decomposition of $f,\left\{P_{i}\right\}_{i=1}^{k}$, for which $\left\|P_{i}\right\|>r^{\prime}$ for each $i=1,2, \cdots, k$.

Proof. The lemma is easily established by a straightforward construction if each member of $F(f, r)$ is open. Hence we assume that $G$ is a member of $F(f, r)$ which is not open. Let

$$
A_{0}=\bigcap\{A: A \text { open and closed in } B(f) \text { and } G \subset A\} .
$$

Now $A_{0}$ cannot be open: for if it were, any nonempty set, open and closed in $A_{0}$, would be open and closed in $B(f)$, and hence would be $A_{0}$. This would imply that $A_{0}$ was connected, and we arrive at the contradiction that $A_{0}$ is the nonopen set $G$.

Now let $x_{0}$ be in $A_{0}$-int $A_{0}$. Let $r^{\prime}<\left|f\left(x_{0}\right)\right|$. There is a neighborhood $N_{0}$ of $x_{0}$ throughout which $|f(x)|>r^{\prime}$. Choose $x_{1}$ in $N_{0}$ - $\left(N_{0} \cap A_{0}\right)$. There exists an $A_{1}$ open and closed in $B(f)$ such that $A_{0} \subset A_{1}$, and $x_{1}$ is in $B_{1}=B(f)-A_{1}$. Now $N_{0} \cap A_{1}$ is also a neighborhood of $x_{0}$. Choose $x_{2}$ in $\left(N_{0} \cap A_{1}\right)-\left(N_{0} \cap A_{0}\right)$ and find $A_{2}$ open and closed in $B(f)$ for which $A_{0} \subset A_{2} \subset A_{1}$, and $x_{2}$ is in $B_{2}=B(f)-A_{2}$. Continuing this process we obtain $k$ disjoint sets $A_{k-1}, B_{k-1} \cap A_{k-2}, \cdots$, $B_{2} \cap A_{1}, B_{1}$. Each is open and closed in $B(f)$, and each contains a point at which $|f|>r^{\prime}$. These sets may be taken as the supports of $P_{1}, \cdots, P_{k}$ in the statement of the lemma. The proof is completed.

6. Lemma. The function $f$ is well behaved if and only if for each $r>0, F(f, r)$ contains at most a finite number of nonempty sets.

7. Lemмa. If $f$ is well behaved, then for each $r>0$ there is a unique 
decomposition $\left\{P_{i}\right\}_{i=1}^{n}$ of $f$ such that $\left\|P_{n}\right\|<r^{m}$ and for $i=1,2, \cdots, n-1$ both $f_{i}$ admits no proper decomposition, and $\left\|P_{n}\right\|>r$. Furthermore the number of nonempty sets in $F(f, r)$ is $n-1$.

8. LEмma. Let $\left\{P_{i}\right\}_{i=1}^{m}$ be a decomposition of a well behaved function $f$. Let the number of nonempty sets in $F(f, r)$ be $n-1$. There do not exist $n$ distinct values of $i$ such that $\|P\|>r$.

It had seemed reasonable that Lemma 5 above could be replaced by the following stronger statement: There exist $k$ nonempty sets in $F(f, r)$ if and only if there is a decomposition of $f,\left\{P_{i}\right\}_{i=1}^{k}$ such that $\left\|P_{i}\right\|>r$ for $i=1, \cdots, k$. Professor A. J. Ward presented the following counterexample.

9. EXAmple (A. J. Ward). Let $X=\{(x, y): x$ real, $y=1-1 / n, n$ $=2,3, \cdots\} \cup\{(x, y): x$ integral, $1 \leqq y \leqq 2\}$. Let $f(x, y)=y$. Hence $B(f)=X$. The counterexample is obtained by taking $r=3 / 2$.

10. ThEOREM. The only locally connected compactification of $[0,1]$ is $[0,1]$.

Proof. Suppose $f$ is in $C([0,1))$, but $\lim _{x \rightarrow 1} f(x)$ does not exist. Let

$$
2 c=\limsup _{x \rightarrow 1} f(x)+\liminf _{x \rightarrow 1} f(x) .
$$

Now $g(x)=f(x)-c$ is not well behaved. Moreover if $K X$ is a compactification of $X$ such that $g$ has an extension to a continuous function $g^{\prime}$ in $C(K X)$ then $g^{\prime}$ is not well behaved in $C(K X)$.

\section{REFERENCES}

1. B. Banaschewski, Local connectedness of extension spaces, Canad. J. Math. 8 (1956), 385-388.

2. J. DeGroot and R. H. McDowell, Locally connected spaces and their compactifications, Illinois J. Math. 11 (1967), 353-364.

3. J. Dugundji, Topology, Allyn and Bacon, Boston, Mass., 1966.

4. L. Gillman and M. Jerison, Rings of continuous functions, Van Nostrand, Princeton, N. J., 1960.

5. M. Henriksen and J. R. Isbell, Local conrectedness in the Stone-Cech com pactification, Illinois J. Math. 1 (1957), 574-582.

UNIVERSITY OF WASHINGTON 